Research Article

\title{
Impact of Fusion Temperature on Hydrothermal Features of Flow within an Annulus Loaded with Nanoencapsulated Phase Change Materials (NEPCMs) during Natural Convection Process
}

\author{
Seyyed Masoud Seyyedi $\mathbb{D}^{1},{ }^{1}$ M. Hashemi-Tilehnoee $\mathbb{D}^{1},{ }^{1}$ and $M$. Sharifpur $\mathbb{D}^{2,3,4}$ \\ ${ }^{1}$ Department of Mechanical Engineering, Islamic Azad University, Aliabad Katoul Branch, Aliabad Katoul, Iran \\ ${ }^{2}$ Department of Mechanical Engineering, University of Science and Culture, Tehran, Iran \\ ${ }^{3}$ Department of Mechanical and Aeronautical Engineering, University of Pretoria, Pretoria 0002, South Africa \\ ${ }^{4}$ Department of Medical Research, China Medical University Hospital, China Medical University, Taichung, Taiwan \\ Correspondence should be addressed to Seyyed Masoud Seyyedi; s.masoud_seyedi@aliabadiau.ac.ir and M. Sharifpur; \\ msharifpur@usc.ac.ir
}

Received 14 May 2021; Accepted 26 October 2021; Published 10 November 2021

Academic Editor: SIvasankaran Sivanandam

Copyright ( 2021 Seyyed Masoud Seyyedi et al. This is an open access article distributed under the Creative Commons Attribution License, which permits unrestricted use, distribution, and reproduction in any medium, provided the original work is properly cited.

\begin{abstract}
A new type of nanofluids is nanoencapsulated phase change materials (NEPCMs), where nanoparticles are made of a shell and a core. In the current study, characteristics of free convection flow, entropy generation, and heat transfer of NEPCMs in an enclosure are investigated. The enclosure is an annulus between concentric horizontal circular and square cylinders with a porous medium. The governing equations (i.e., continuity, energy, and momentum) are written in the nondimensional form and then numerically solved by the control volume finite element method (CVFEM). The results of the validation are in good agreement with those of the literature. The effects of decision variables on the entropy generation number and the average Nusselt number are investigated. The outcomes discovered that there is a maximum for $\mathrm{Nu}_{\text {ave }}$ and a minimum for $N_{\text {gen }}$ at $\theta_{f}=0.4$ for each value of the Stefan number. Also, $\mathrm{Nu}_{\mathrm{ave}}$ and ECOP increase by $8.8 \%$ and $24.8 \%$, respectively, while $N_{\text {gen }}$ decreases by $12.8 \%$ when $\phi$ increases from 0 (pure fluid) to 0.05 at $\theta_{f}=0.4$.
\end{abstract}

\section{Introduction}

Nowadays, nanofluids are used to enhance heat transfer in many various industries. Simulation of their behavior in enclosures is an interesting subject for researchers because of its many industrial applications.

The literature review shows that there are many studies about natural convection analysis in enclosures [1-8]. As we know, a considerable amount of energy can be stored by a small amount of Phase Change Materials (PCMs) during a solid to liquid phase change process. PCMs can be used in many industrial applications such as cooling electronic devices $[9,10]$, solar heating systems $[11,12]$, waste heat recovery $[13,14]$, and thermal energy storage systems $[15,16]$. But their disadvantage is low thermal conductivity, and therefore, many researchers have tried to improve their thermal conductivity. Recently, they proposed nanoencapsulated phase change materials (NEPCMs). NEPCMs are a new category of nanofluids that nanoparticle includes a shell and a PCM core [17]. In 2015, NEPCM force convection of slurry was studied by Lu et al. [18]. They considered flow on a backward-facing step and investigated the effect of Reynolds number, wall heat flux, and nanoparticle volume fraction on heat transfer and flow. In 2015 and 2018, two good review papers about NEPCMs were published by Liu et al. [19] and Chai et al. [20], respectively. The former investigated the increment of heat transfer, methods of preparation, and characterization, while the last focused on the study of mini/microchannel heat sinks with nano/ microencapsulated phase change slurry as working fluid. In 
2019, Ghalambaz et al. [21] investigated the thermal behavior of NEPCMs in a porous medium over a vertical flat plate. They discovered that the Nusselt number increases when the fusion temperature of the NEPCMs decreases. In 2019, Ahmad et al. [22] investigated the transient free convection in a NEPCM. They concluded that the heat transfer is affected by the variation of the PCM core fusion temperature. In 2019, the behavior of NECPMs in a square enclosure was investigated by Ghalambaz et al. [17]. They concluded that the fusion temperature is an important variable that has a major effect on heat transfer. Also, in 2019 , they conducted an unsteady free convection flow of NEPCMs in a porous medium [23]. In 2020, the influence of $n$th order chemical reaction in an unsteady nanofluid bioconvective channel flow was investigated by Basir et al. [24]. In 2020, the natural convection flow of a suspension containing nanoencapsulated phase change particles in an eccentric annulus was investigated by Mehryan et al. [25]. In 2020, Ho et al. [26] studied forced convection heat transfer of NEPCM suspension in a minichannel heatsink. In 2020, thermohydrodynamic and irreversibility analyses for NEPCMs were performed by Hashem Zadeh et al. [27, 28].

The literature review shows that the second law analysis has not been performed for an enclosure filled by NEPCMs. In this work, the entropy generation and natural convection analyses are performed in an annulus between square cylinders and concentric horizontal circular filled with NEPCMs.

The characteristics of this paper in comparison with the previous works are as follows:

(1) The geometry is a complex enclosure with a porous medium

(2) The nanoparticles are NEPCM that are dispersed in the base fluid

(3) The effects of important parameters on Nusselt number are studied

(4) The entropy generation number is calculated

(5) The thermal performance of the enclosure is evaluated by a criterion known as the ecological coefficient of performance (ECOP)

\section{Problem Statements}

2.1. Physical Model. A schematic view of the problem is shown in Figure 1. The system consists of a heated horizontal cylinder that is concentrically placed inside a square enclosure with a porous medium. The outer square and inner circle are held at fixed temperatures $T_{c}$ and $T_{h}\left(T_{c}<T_{h}\right)$, respectively.

\subsection{Mathematical Model}

2.2.1. Basic Governing Equations. The natural convective flow is assumed to be two-dimensional, steady, laminar, and incompressible. Also, it is assumed that the variation of fluid properties and the radiation effects are negligible. Albeit, the variations of density in the buoyancy term are considered where the Boussinesq approximation is used. The core of
NEPCM undergoes a phase change at the fusion temperature of $T_{f}$ where $T_{c}<T_{f}<T_{h}$. Thus, the governing equations can be written as follows $[6,17]$ :

$$
\begin{aligned}
& \frac{\partial u}{\partial x}+\frac{\partial v}{\partial y}= 0 \\
& \rho_{b}\left(u \frac{\partial u}{\partial x}+v \frac{\partial u}{\partial y}\right)=-\frac{\partial P}{\partial x}+\mu_{b}\left(\frac{\partial^{2} u}{\partial x^{2}}+\frac{\partial^{2} u}{\partial y^{2}}\right)-\frac{\mu_{b}}{K} u \\
& \rho_{b}\left(u \frac{\partial v}{\partial x}+v \frac{\partial v}{\partial y}\right)=-\frac{\partial P}{\partial y}+\mu_{b}\left(\frac{\partial^{2} v}{\partial x^{2}}+\frac{\partial^{2} v}{\partial y^{2}}\right)+g \rho_{b} \beta_{b}\left(T-T_{c}\right) \\
&-\frac{\mu_{b}}{K} v \\
&\left(\rho C_{p}\right)_{b}\left(u \frac{\partial T}{\partial x}+v \frac{\partial T}{\partial y}\right)=k_{b}\left(\frac{\partial^{2} T}{\partial x^{2}}+\frac{\partial^{2} T}{\partial y^{2}}\right)
\end{aligned}
$$

2.2.2. Governing Equations in Nondimensional Form. The vorticity $(\omega)$, stream function $(\psi)$, and dimensionless variables are as follows:

$$
\begin{aligned}
u & =\frac{\partial \psi}{\partial y}, \\
v & =-\frac{\partial \psi}{\partial x}, \\
\omega & =\frac{\partial v}{\partial x}-\frac{\partial u}{\partial y}, \\
X & =\frac{x}{L}, \\
Y & =\frac{y}{L}, \\
A R & =\frac{R}{L}, \\
\theta & =\frac{T-T_{c}}{T_{h}-T_{c}}, \\
\Omega & =\frac{\omega L^{2}}{\alpha_{f}}, \\
\Psi & =\frac{\psi}{\alpha_{f}} .
\end{aligned}
$$

Thus, using equations (5) and (6), the nondimensional form of equations (1)-(4) can be rewritten as

$$
\begin{aligned}
\frac{\partial^{2} \Psi}{\partial X^{2}}+\frac{\partial^{2} \Psi}{\partial Y^{2}}= & -\Omega, \\
{\left[\frac{\partial \Psi}{\partial Y} \frac{\partial \Omega}{\partial X}-\frac{\partial \Psi}{\partial X} \frac{\partial \Omega}{\partial Y}\right]=} & \frac{\mu_{b} / \mu_{f}}{\rho_{b} / \rho_{f}} \operatorname{Pr}\left(\frac{\partial^{2} \Omega}{\partial X^{2}}+\frac{\partial^{2} \Omega}{\partial Y^{2}}\right) \\
& +\frac{\beta_{b}}{\beta_{f}} \operatorname{Ra} \operatorname{Pr} \frac{\partial \theta}{\partial X}-\frac{\mu_{b} / \mu_{f}}{\rho_{b} / \rho_{f}} \frac{\operatorname{Pr}}{\mathrm{Da}} \Omega,
\end{aligned}
$$



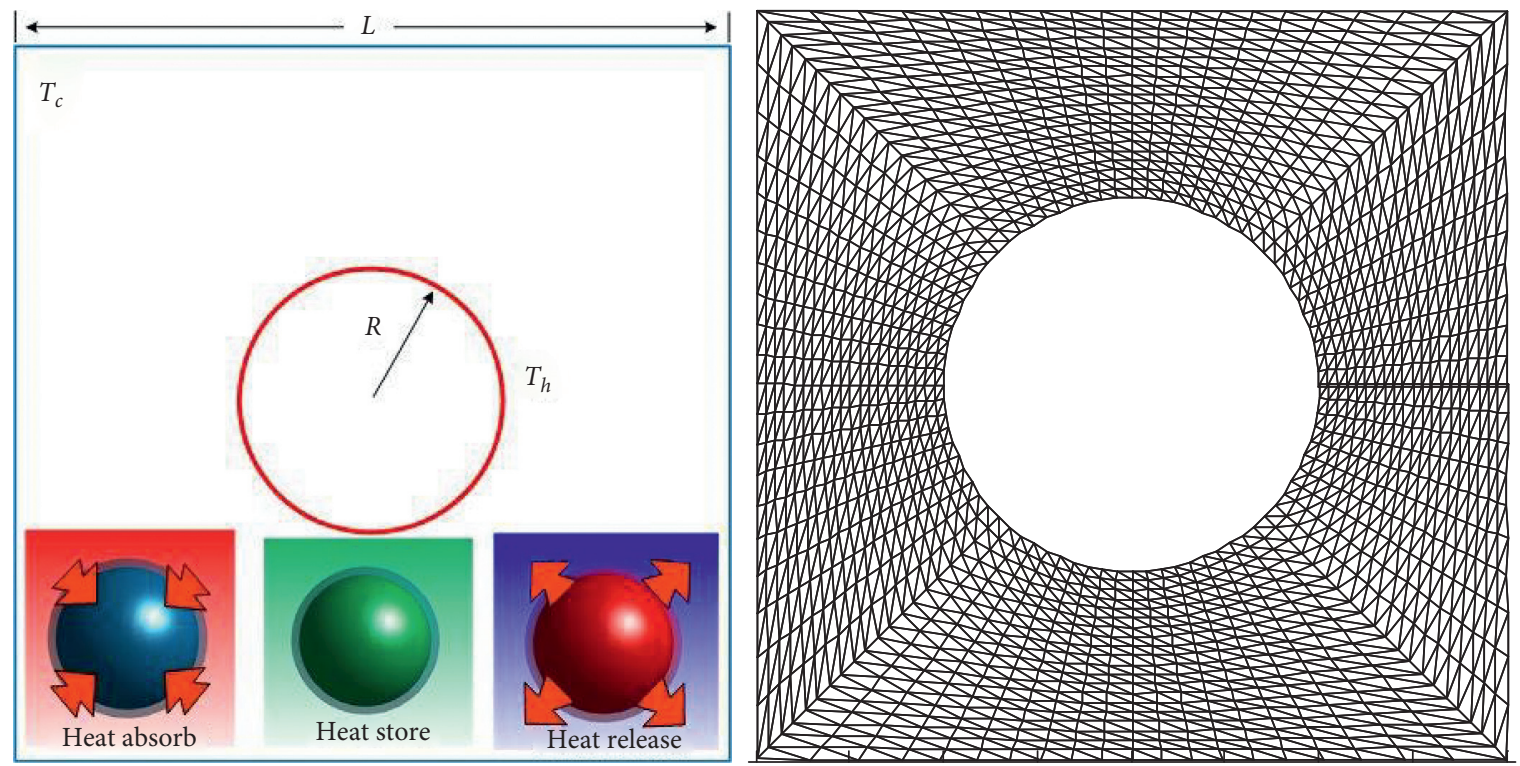

FIGURE 1: Schematic view of the considered problem.

$$
\begin{aligned}
{\left[\frac{\partial \Psi}{\partial Y} \frac{\partial \theta}{\partial X}-\frac{\partial \Psi}{\partial X} \frac{\partial \theta}{\partial Y}\right] } & =\frac{k_{b} / k_{f}}{\operatorname{Cr}}\left(\frac{\partial^{2} \theta}{\partial X^{2}}+\frac{\partial^{2} \theta}{\partial Y^{2}}\right), \\
\operatorname{Ra} & =\frac{g \beta_{f} L^{3}\left(T_{h}-T_{c}\right)}{\nu_{f} \alpha_{f}}, \\
\operatorname{Pr} & =\frac{\nu_{f}}{\alpha_{f}}, \\
\operatorname{Da} & =\frac{K}{L^{2}}, \\
\mathrm{Cr} & =\frac{\left(\rho C_{p}\right)_{b}}{\left(\rho C_{p}\right)_{f}}
\end{aligned}
$$

In the previous equation, $\lambda, \delta$, and Ste are the ratio of the heat capacity of the NEPCM nanoparticles to the base fluid, the nondimensional parameter of fusion range, and Stefan number, respectively. For more details, see [17]. Also, $f$ is the nondimensional fusion function defined by the following equation:

$$
f=\frac{\pi}{2} \sin \left(\frac{\pi}{\delta}\left(\theta-\theta_{f}+\frac{\delta}{2}\right)\right) \times \begin{cases}0 & \theta<\theta_{f}-\frac{\delta}{2} \\ 1 & \theta_{f}-\frac{\delta}{2}<\theta<\theta_{f}+\frac{\delta}{2} . \\ 0 & \theta_{f}+\frac{\delta}{2}<\theta\end{cases}
$$

The boundary conditions corresponding to Figure 1 are

$$
\begin{aligned}
\theta & =1.0 \text { on the inner boundary, } \\
\theta & =0.0 \text { on the outer boundary, } \\
\Psi & =0 \text { on all solid boundaries. }
\end{aligned}
$$

$\beta_{b}=(1-\phi) \beta_{f}+\phi \beta_{p}$

$\frac{k_{b}}{k_{f}}=1+N c \phi$

$$
\frac{\mu_{b}}{\mu_{f}}=1+N v \phi
$$

The heat capacity ratio $(\mathrm{Cr})$ can be written in the nondimensional form as [17]

$$
C r=\frac{\left(\rho C_{p}\right)_{b}}{\left(\rho C_{p}\right)_{f}}=(1-\phi)+\phi \lambda+\frac{\phi}{\delta S t e} f .
$$

2.2.3. Entropy Generation. The rate of entropy generation can be computed as follows: 


$$
\begin{aligned}
\dot{S}_{\text {gen }}= & \frac{k_{b f}}{T_{0}^{2}}\left[\left(\frac{\partial T}{\partial x}\right)^{2}+\left(\frac{\partial T}{\partial y}\right)^{2}\right]+\frac{\mu_{b f}}{T_{0}}\left[2\left(\frac{\partial u}{\partial x}\right)^{2}+2\left(\frac{\partial v}{\partial y}\right)^{2}\right. \\
& \left.+\left(\frac{\partial u}{\partial y}+\frac{\partial v}{\partial x}\right)^{2}\right]+\frac{\mu_{b}\left(u^{2}+v^{2}\right)}{K T_{0}} .
\end{aligned}
$$

The local entropy generation in a nondimensional form can be written as follows [6]:

$$
\begin{aligned}
N_{L, \text { gen }}= & \frac{\dot{S}_{\text {gen }}}{\left[\left(k_{f} / T_{0}^{2}\right)(\Delta T / L)^{2}\right]}=\frac{k_{b}}{k_{f}}\left[\left(\frac{\partial \theta}{\partial X}\right)^{2}+\left(\frac{\partial \theta}{\partial Y}\right)^{2}\right] \\
& +\frac{\mu_{b}}{\mu_{f}} \Phi_{f}\left[4\left(\frac{\partial^{2} \Psi}{\partial X \partial Y}\right)^{2}+\left(\frac{\partial^{2} \Psi}{\partial Y^{2}}-\frac{\partial^{2} \Psi}{\partial X^{2}}\right)^{2}\right] \\
& +\frac{1}{\mathrm{Da}} \frac{\mu_{b}}{\mu_{f}} \Phi_{f}\left[\left(\frac{\partial \Psi}{\partial X}\right)^{2}+\left(\frac{\partial \Psi}{\partial Y}\right)^{2}\right], \\
\Phi_{f}= & \frac{\mu_{f} T_{0}}{k_{f}}\left(\frac{\alpha_{f}}{L \Delta T}\right)^{2},
\end{aligned}
$$

where $\left(T_{0}=\left(T_{h}+T_{c}\right) / 2\right)$ is the mean temperature and $\Delta T$ is the temperature difference within the enclosure $\left(\Delta T=T_{h}-T_{c}\right)$.

Equation (22) can be rewritten as follows:

$$
\begin{aligned}
& N_{L, \mathrm{gen}}=N_{L, \mathrm{HT}}+N_{L, \mathrm{FF}}+N_{L, \mathrm{PM}}, \\
& N_{L, \mathrm{HT}}=\frac{k_{b}}{k_{f}}\left[\left(\frac{\partial \theta}{\partial X}\right)^{2}+\left(\frac{\partial \theta}{\partial Y}\right)^{2}\right], \\
& N_{L, \mathrm{FF}}=\frac{\mu_{b}}{\mu_{f}} \Phi_{f}\left[4\left(\frac{\partial^{2} \Psi}{\partial X \partial Y}\right)^{2}+\left(\frac{\partial^{2} \Psi}{\partial Y^{2}}-\frac{\partial^{2} \Psi}{\partial X^{2}}\right)^{2}\right], \\
& N_{L, \mathrm{PM}}=\frac{1}{\mathrm{Da}} \frac{\mu_{b}}{\mu_{f}} \Phi_{f}\left[\left(\frac{\partial \Psi}{\partial X}\right)^{2}+\left(\frac{\partial \Psi}{\partial Y}\right)^{2}\right] .
\end{aligned}
$$

The total entropy generation can be obtained by

$$
\begin{aligned}
N_{T, \mathrm{HT}} & =\int_{V} N_{L, \mathrm{HT}} \mathrm{d} V, \\
N_{T, \mathrm{FF}} & =\int_{V} N_{L, \mathrm{FF}} \mathrm{d} V, \\
N_{T, \mathrm{PM}} & =\int_{V} N_{L, \mathrm{PM}} \mathrm{d} V .
\end{aligned}
$$

The entropy generation number can be calculated by

$$
N_{\text {gen }}=N_{T, \mathrm{FF}}+N_{T, \mathrm{HT}}+N_{T, \mathrm{PM}} .
$$

2.2.4. Ecological Coefficient of Performance (ECOP). It is known as the ecological coefficient of performance (ECOP) proposed by Seyyedi et al. [4] is a criterion for the evaluation of enclosure thermal performance:

$$
\mathrm{ECOP}=\frac{\mathrm{Nu}_{\text {ave }}}{N_{\text {gen }}}
$$

\section{Numerical Procedure}

A FORTRAN code has been developed using the control volume finite element method (CVFEM) to solve the governing equations.

3.1. Grid Test. The results of the grid independence study are provided in Table 1. The calculations have been performed with $\mathrm{Ra}=10^{5}, \mathrm{Da}=0.01, \operatorname{Pr}=6.2, \phi=0.05, \delta=0.05$, Ste $=0.313, \theta_{f}=0.3, \lambda=0.4, \mathrm{AR}=0.2$, and $\Phi=10^{-4}$. Hence, $111 \times 481$ can be considered as the grid size.

3.2. Validation. For validation of the written code, it is validated by comparison of the outcomes with the published results in the literature. Table 2 shows the values of the average Nusselt number on the hot surface (inner circular in Figure 1), for $\mathrm{Pr}=0.71$ at $\mathrm{Ra}=10^{4}$ and $10^{5}$. A good agreement can be seen between the present results and those of previous works. Also, Figure 2 depicts the isothermals and streamlines of the literature and present work at $\mathrm{Ra}=10^{5}$ corresponding to Table 2 .

Furthermore, another validation has been provided for the isotherms, streamlines, and Cr contour for $\theta_{f}=0.3$ at $\mathrm{Ra}=10^{5}$. Figure 3 presents a comparison of the results between Ghalambaz et al. [17] and presents work that is acceptable.

\section{Results and Discussion}

In this work, the entropy generation and the free convection heat transfer in an annulus between concentric horizontal circular and square cylinders loaded with a dilute suspension are numerically investigated by CVFEM. The effects of nondimensional parameters on the characteristics of flow and entropy generation number are investigated. Table 3 presents the default values of the nondimensional parameters for calculations unless the new values are stated.

Figure 4 shows the streamlines, the isotherms, and $\mathrm{Cr}$ contours at $\mathrm{Ra}=10^{4}, 10^{5}$, and $10^{6}$. There are two overall rotating symmetric eddies with two inner vortices at $\mathrm{Ra}=10^{4}$, but when Rayleigh number goes up to $10^{5}$ and $10^{6}$, the recirculating eddies core is placed only at the upper half. In other words, the circulation of the flow increased with increasing the Rayleigh number. The maximum absolute value of the stream function, $\left|\Psi_{\max }\right|$, increases when the Rayleigh number increases. The values of $\left|\Psi_{\max }\right|$ are 0.804 , 
TAble 1: The effect of grid size on $\Psi_{\text {max }}, \mathrm{Nu}_{\text {ave }}$, and the $N_{\text {gen }}$.

\begin{tabular}{lcccccc}
\hline & $31 \times 361$ & $51 \times 381$ & $71 \times 401$ & $91 \times 421$ & $111 \times 481$ & 6.21 \\
\hline$\Psi_{\max }$ & 6.5 & 6.33 & 6.26 & 6.22 & $5.121 \times 521$ \\
$\mathrm{Nu}_{\text {ave }}$ & 5.0045 & 5.1741 & 5.1834 & 5.1859 & 5.1853 & 5.1852 \\
$N_{\text {gen }}$ & 50.02 & 51.01 & 51.63 & 51.94 & 52.13 & 52.15 \\
\hline
\end{tabular}

TABLE 2: Comparison of the average Nusselt number $\left(\mathrm{Nu}_{\mathrm{ave}}\right)$ on the inner circular for the present study with previous numerical studies for $A R=R / L=0.2$ and $\operatorname{Pr}=0.71$.

\begin{tabular}{lccc}
\hline $\mathrm{Ra}$ & Moukalled and Acharya [29] & Kim et al. [30] & Present study \\
\hline $10^{4}$ & 3.331 & 3.414 & 3.339 \\
$10^{5}$ & 5.08 & 5.1385 & 5.1021 \\
\hline
\end{tabular}

6.81, and 21.64 for $\mathrm{Ra}=10^{4}, 10^{5}$, and $10^{6}$, respectively. As it can be seen from the isotherms, for $\mathrm{Ra}=10^{4}$, the heat transfer in the cavity is mainly dominated by the conduction mode, whereas the heat transfer in the enclosure is mainly dominated by the convection mode at $\mathrm{Ra}=10^{6}$. The role of convection in heat transfer becomes more significant with increasing the Rayleigh number, and the thermal boundary layer on the hot wall becomes thinner. The average Nusselt number ascends with ascending the Rayleigh number where the values of $\mathrm{Nu}_{\text {ave }}$ are 4.1636, 6.0981, and 10.962, for $\mathrm{Ra}=10^{4}, 10^{5}$, and $10^{6}$, respectively. The last column in Figure 4 presents the $\mathrm{Cr}$ contours for each Rayleigh number. According to equations (16) and (17), Cr has a minimum value that is corresponding to $f=0$. (other parameters are assumed to be constant). For example, for $\phi=0.05, \lambda=0.04$, $\delta=0.05$, and Ste $=0.313$, the minimum value of $\mathrm{Cr}$ is 0.97 (when $f=0$ ). Also, $\mathrm{Cr}$ has a maximum value that depends on $\theta_{f}$ in the $f$ correlation. Here, the maximum value of $\mathrm{Cr}$ is 8.82 (this value can be obtained by solving the velocity and temperature fields). The values of 0.97 and 8.82 are the same for each value of the Rayleigh number in Figure 4. Notice that the heat capacity changes since the phase change has occurred.

Figure 5 presents the entropy generation number, the average Nusselt number, and ECOP versus the fusion temperature of the NEPCM core $\left(\theta_{f}\right)$ for three values of the Stefan number at $\mathrm{Ra}=10^{5}$ and $\phi=0.05$. Figure 5(a) shows that there is a maximum value for $\mathrm{Nu}_{\mathrm{ave}}$ at $\theta_{f}=0.4$ for each value of the Stefan number. Albeit, it can be stated that the variations of the average Nusselt number are very small in the range of $0.3<\theta_{f}<0.7$ for each value of the Stefan number. Also, the average Nusselt number decreases with increasing the Stefan number for a constant value of $\theta_{f}$. It should be noted that the Stefan number increases as the PCM cores latent heat decreases. Also, the heat storage capacity of the NEPCM particles descends with descending the PCM cores latent heat. Finally, the convective heat transfer rate in the cavity diminishes when the heat storage capacity of the NEPCM particles decreases. Figure 5(b) demonstrates that the entropy generation number firstly decreases, reaches a minimum value, and then goes up as the fusion temperature increases for each value of the Stefan number. Also, decreasing the values of Stefan number is desirable from the second law of thermodynamics point of view since the entropy generation number decreases when the Stefan number decreases for each value of $\theta_{f}$. Figure 5(c) illustrates a maximum value for ECOP when it is plotted versus $\theta_{f}$ for each Stefan number. Therefore, Figure 5 shows that $\mathrm{Nu}_{\text {ave }}$ and ECOP increase whereas $N_{\text {gen }}$ decreases as Ste decreases. For example, $\mathrm{Nu}_{\text {ave }}$ increases from 5.1135 to 5.3484 (4.6\% increase) and ECOP increases from 0.0971 to 0.1070 (10.2\% increase) while $N_{\text {gen }}$ decreases from 52.67 to 50.00 (5.3\% decrease) when Ste decreases from 0.5 to 0.2 at $\theta_{f}=0.4$.

Figure 6 presents the ECOP, the entropy generation number, and the average Nusselt number versus the volume fraction of the nanoparticle for three values of fusion temperature. As could be seen, raising the volume fraction of nanoparticle is desirable since $\mathrm{Nu}_{\mathrm{ave}}$ and ECOP rise and $N_{\text {gen }}$ descends with raising $\phi$ for $\theta_{f}$. For instance, $\mathrm{Nu}_{\text {ave }}$ increases from 4.7911 to 5.2148 ( $8.8 \%$ increase) and ECOP increases from 0.0812 to 0.1013 (24.8\% increase) while $N_{\text {gen }}$ decreases from 58.99 to 51.46 (12.8\% decrease) when $\phi$ increases from 0 (pure fluid) to 0.05 at $\theta_{f}=0.4$. On the contrary, Figure 6 shows that the variations of the entropy generation number, the average Nusselt number, and ECOP is small when the nondimensional fusion temperature varies from 0.2 to 0.4 . For another example, $\mathrm{Nu}_{\text {ave }}$ increases from 4.9915 to 5.0534 (1.2\% increase) and ECOP increases from 0.0906 to 0.0935 ( $3.2 \%$ increase) while $N_{\text {gen }}$ decreases from 55.10 to 54.07 (1.9\% decrease) when $\theta_{f}$ increases from 0.02 to 0.04 at $\phi=0.03$.

Figure 7 presents $\mathrm{Nu}_{\text {ave }}, N_{\text {gen }}$, and ECOP versus $\mathrm{Da}$ for three values of Ste at $\theta_{f}=0.4$ and $\mathrm{Ra}=10^{5}$. The result demonstrates that $\mathrm{Nu}_{\text {ave }}, N_{\text {gen }}$, and ECOP ascend with increasing $\mathrm{Da}$ for Ste. For example, $\mathrm{Nu}_{\text {ave }}$ ascends from 5.1135 to 5.3853 ( $5.3 \%$ increase), $N_{\text {gen }}$ enhances from 52.67 to 53.93 (2.4\% increase), and ECOP increases from 0.0971 to 0.0999 ( $2.9 \%$ increase) when Darcy number goes up from 0.01 to 100 at Ste $=0.5$.

Figure 8 depicts the streamlines, the isotherms, and the $\mathrm{Cr}$ contours for different values of aspect ratio (0.1, 0.2 and 0.3 ) at $\theta_{f}=0.45$ and $\mathrm{Ra}=10^{5}$. The streamlines show that the flow circulation decreases with increasing the aspect ratio values. In other words, the strength of the flow (indicated by $\left|\Psi_{\max }\right|$ ) decreases as the values of the aspect ratio go up. Its reason can be the fact that the available space for flow circulation descends when the aspect ratio ascends. On the contrary, $\mathrm{Nu}_{\text {ave }}$ increases with increasing aspect ratio values because of the increased heat transfer surface area associated with higher $A R$ values. Also, $\mathrm{Nu}_{\text {ave }}$ increases since the space between the walls decreases with increasing the values of aspect ratio. The isotherms follow the shapes of inner and outer walls near the hot and cold walls, respectively. At $A R=0.1$, the 

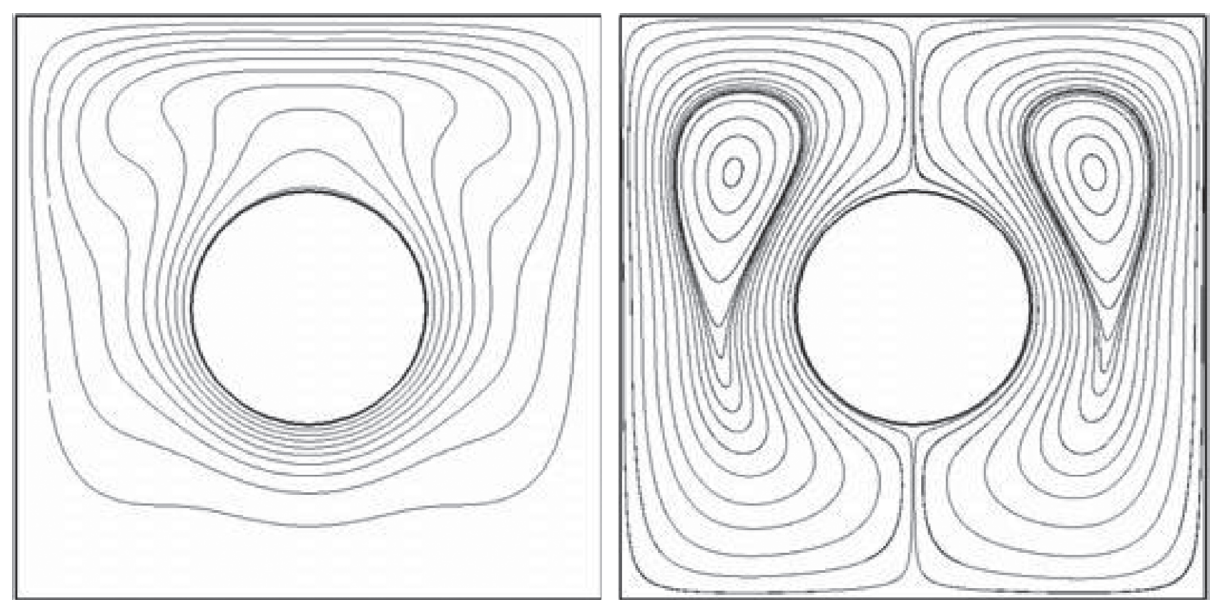

(a)
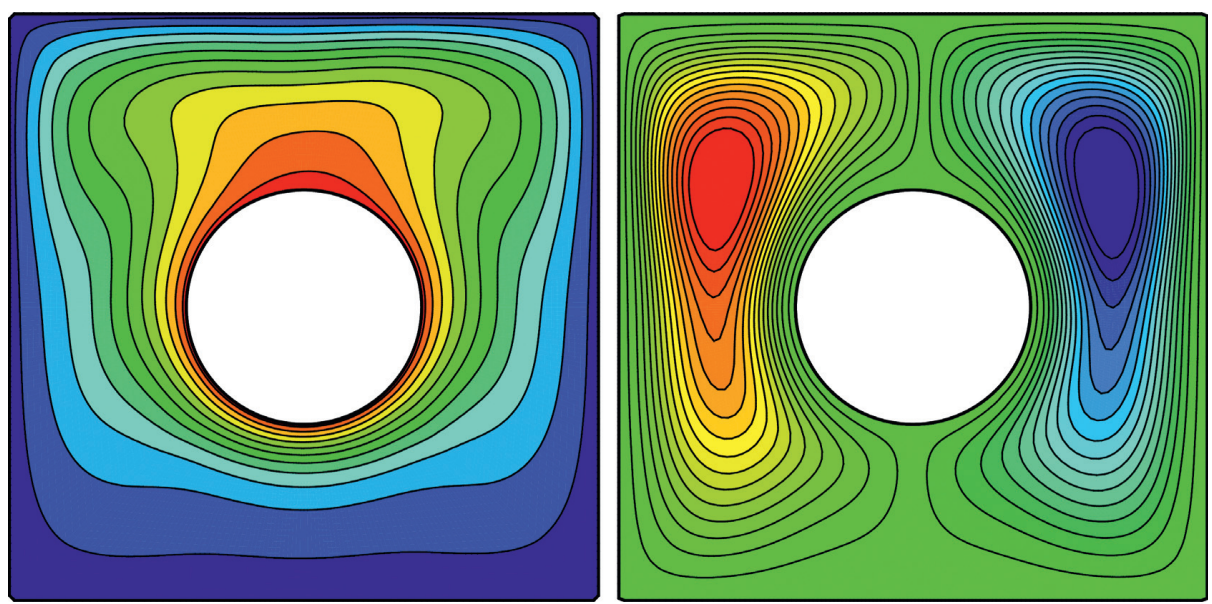

(b)

Figure 2: The isotherms (left) and streamlines (right) at $\mathrm{Ra}=10^{5}$ for (a) Kim et al. [30] and (b) the present work.

boundary layer along the hot wall separates from the surface near the cylinder top and constructs a thermal plume. The last row of Figure 8 presents the $\mathrm{Cr}$ contours where the color region (except for the blue color region) illustrates the phase change region. The value of $\mathrm{Cr}$ is 0.97 (outside the fusion region corresponding to $\phi=0.05$ ) while it changes inside the fusion region where it has a maximum value of 5.99 in Figure 8 for all aspect ratios. The $\mathrm{Cr}$ contour is similar to a plume at $A R=0.1$, and it is similar to a ring at $A R=0.3$. It shows that the fusion region is near to the bottom of the inner cylinder, and it moves further upward at the top of the inner cylinder for $A R=0.1$. On the contrary, the distance of the fusion region from the inner cylinder and outer wall is approximately the same at $A R=0.3$.

Table 4 presents the effects of aspect ratio on $\left|\Psi_{\max }\right|$, $\mathrm{Nu}_{\text {ave }}$, and $N_{\text {gen }}$ at three values of $\mathrm{Ra}\left(0,10^{4}\right.$ and $\left.10^{5}\right)$. The first row at the second column is corresponding to the value of the average Nusselt number for pure conduction (i.e., $\mathrm{Ra}=0)$. As seen, the values of $\left|\Psi_{\max }\right|, \mathrm{Nu}_{\text {ave }}$, and $N_{\text {gen }}$ increase with increasing the Rayleigh number for each aspect ratio. The increasing in $\left|\Psi_{\max }\right|$ means that the fluid flows more rapidly and increasing $\mathrm{Nu}_{\text {ave }}$ indicates that convection will be the dominant heat transfer mechanism as the Rayleigh number increases. Increasing the value of $N_{\text {gen }}$ is undesirable (from view point of the thermodynamics second law) when Ra increases. At a constant value of Rayleigh number, the values of $\left|\Psi_{\max }\right|$ decrease while the values of $\mathrm{Nu}_{\text {ave }}$ and $N_{\text {gen }}$ increase with increasing the value of AR. For example, $\left|\Psi_{\max }\right|$ descends from 7.558 to 3.740 (50.52\% decrease), $\mathrm{Nu}_{\text {ave }}$ increases from 4.3144 to 7.6795 (78\% increase), and $N_{\text {gen }}$ ascends from 51.6553 to 61.9543 (19.94\% increase) when $A R$ increases from 0.1 to 0.3 at $\mathrm{Ra}=10^{5}$. The last row of Table 4 is corresponding to Figure 8 .

Figure $9(\mathrm{a})$ depicts variations of $\mathrm{Nu}_{\text {local }}$ along the hot wall of the cavity for different values of Stefan number. It has a maximum value at the top of the inner wall $\left(\zeta=90^{\circ}\right)$ for each value of the Stefan number. Figure 9 demonstrates that the difference between the values of the local Nusselt number is very small when the Stefan number increases from 0.2 to 0.5 at each place of the hot wall. The variations of the local Nusselt number along the inner cylinder for different values of Rayleigh number and various values of aspect ratio have been shown in Figures 9(b) and 9(c), respectively. As expected, Figure 9(b) shows that the curve corresponding to $\mathrm{Ra}=10^{5}$ is placed above two other curves. As seen, there is a 

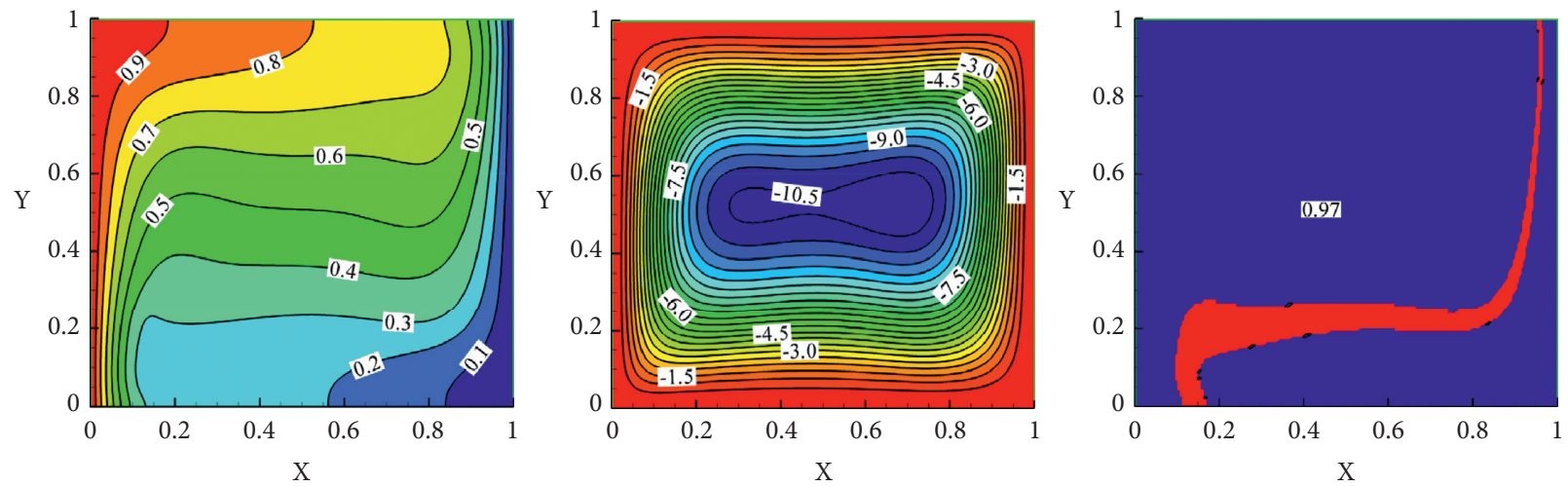

(a)
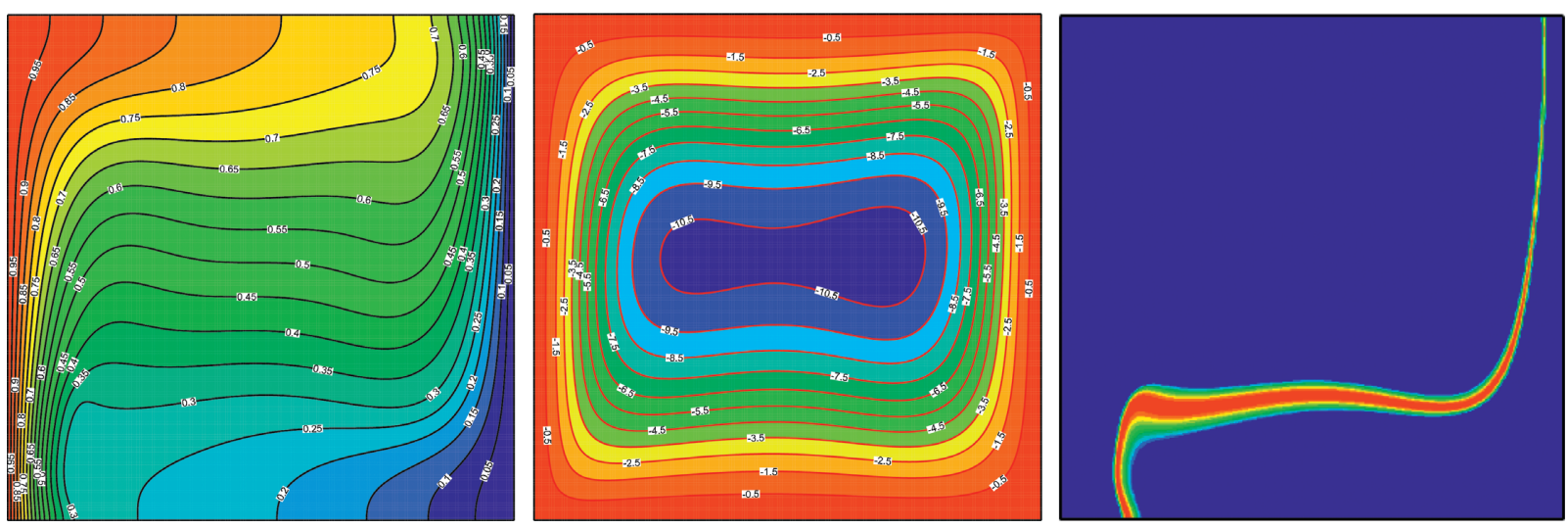

(b)

Figure 3: The isotherms, streamlines, and $\mathrm{Cr}$ contour at $\theta_{f}=0.3$ and $\mathrm{Ra}=10^{5}$ for (a) Ghalambaz et al. [17] and (b) the present study.

TABLe 3: The default values of the nondimensional parameters.

\begin{tabular}{cccccccccccccc}
\hline$A R$ & $\mathrm{Ra}$ & $\mathrm{Da}$ & $\Phi$ & $\operatorname{Pr}$ & $\theta_{f}$ & $\phi$ & $\lambda$ & Ste & $\delta$ & $N_{c}$ & $N_{v}$ & $\rho_{p} / \rho_{f}$ & $\phi \times \beta_{p} / \beta_{f}$ \\
\hline 0.2 & $10^{5}$ & $10-^{2}$ & $10-^{4}$ & 6.2 & 0.3 & 0.05 & 0.4 & 0.313 & 0.05 & 3 & 3 & 0.9 & 0 \\
\hline
\end{tabular}

peak for $\mathrm{Ra}=10^{5}$ at $\zeta=90^{\circ}$ whereas the values of the local Nusselt number approximately are equal to each other at $\zeta=0^{\circ}, 90^{\circ}, 180^{\circ}, 270^{\circ}$, and $360^{\circ}$ for $\mathrm{Ra}=10^{3}$. Figure $9(\mathrm{c})$ shows that the behavior of the local Nusselt number along the hot wall approximately is the same in the range of $45^{\circ}<\zeta<135^{\circ}$ for each aspect ratio. Also, the maximum value of the local Nusselt number decreases as the aspect ratio increases at $\zeta=90^{\circ}$.

Figure 10 demonstrates the $\mathrm{Cr}$ contours for three values of the dimensionless fusion temperature $\left(\theta_{f}=0.1,0.3\right.$, and 0.5$)$. In Figure 10, the red area presents the phase change region in which a phase change occurs. The region with the phase change approaches the cold wall (inner cylinder) when the fusion temperature increases. The red region is similar to a square (cold wall) at $\theta_{f}=0.1$. It is similar to an irregular ring at $\theta_{f}=0.3$, while it is similar to a plume at $\theta_{f}=0.5$. In Figure 10, the values of $\mathrm{Cr}_{\min }$ and $\mathrm{Cr}_{\max }$ are 0.97 and 5.988, respectively. Notice that the value of 0.97 is corresponding to $\phi=0.05$ and $f=0$.
Figure 11(a) presents the contribution of $N_{T, \mathrm{HT}}$ and $N_{\text {T,FF }}$ in the total entropy generation number for an enclosure without porous medium at various values of Rayleigh number. Figure 11 represents the contribution of $N_{T, F F}$ increases as the Ra increases. As evident, the contribution of the $N_{T, \mathrm{HT}}$ increases as the Rayleigh number increases. For example, $N_{T, F F}$ ascends from $4 \%$ to $67 \%$ when the Rayleigh number ascends from $10^{4}$ to $10^{5}$. The reason can be the increasing velocity of fluid flow (or in other words increasing the values of $\left.\left|\Psi_{\max }\right|\right)$ as the Rayleigh number increases. As we know, the friction losses enhance with the enhancement of fluid flow velocity. Figure 11(b) depicts the contribution of $N_{T, \mathrm{HT}}, N_{T, \mathrm{FF}}$, and $N_{T, \mathrm{PM}}$ in the total entropy generation number for two values of volume fraction of nanoparticles $(\phi=0.01$ and 0.05$)$ at two values of the fusion temperature $\left(\theta_{f}=0.2\right.$ and 0.4$)$. As it can be seen, $N_{T, \mathrm{HT}}$ increases when $\phi$ increases from 0.01 to 0.05 whereas $N_{T, F F}$ decreases with ascending the nanoparticles volume fraction for each value of fusion temperature. For example, $N_{T, \mathrm{HT}}$ goes up from 


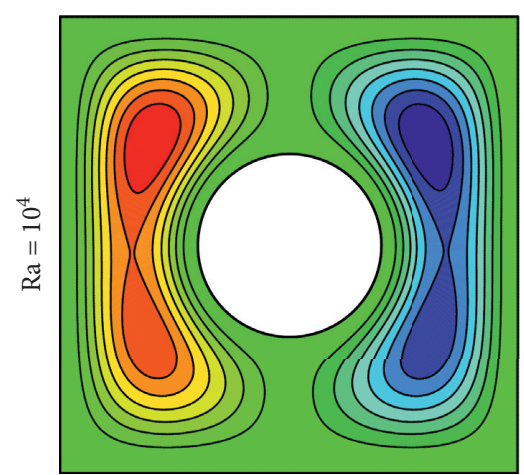

$\left|\Psi_{\max }\right|=0.804$

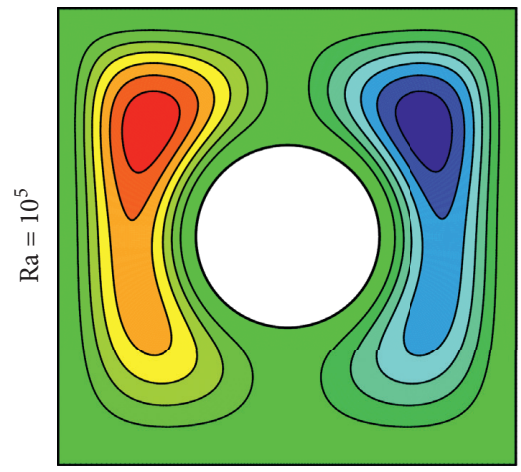

$\left|\Psi_{\max }\right|=6.81$

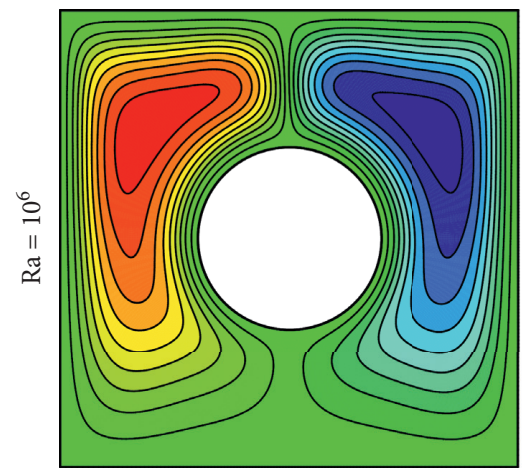

$\left|\Psi_{\text {max }}\right|=21.64$

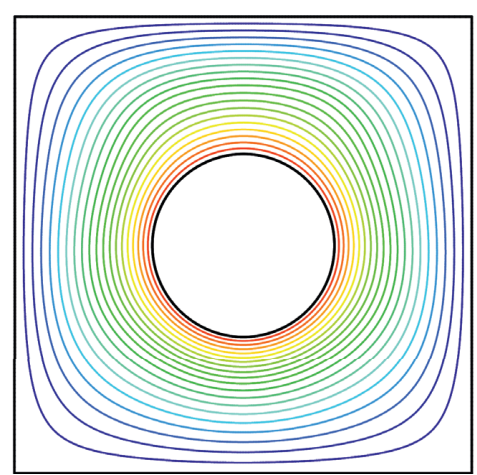

$\mathrm{Nu}_{\text {ave }}=4.1636$

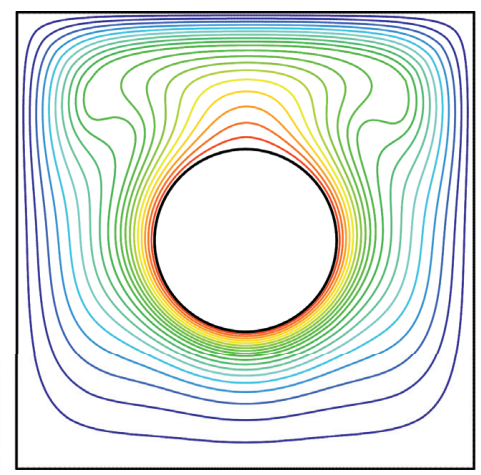

$\mathrm{Nu}_{\mathrm{ave}}=6.0981$

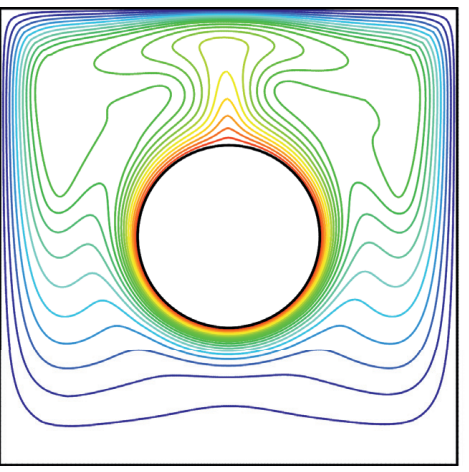

$\mathrm{Nu}_{\text {ave }}=10.962$
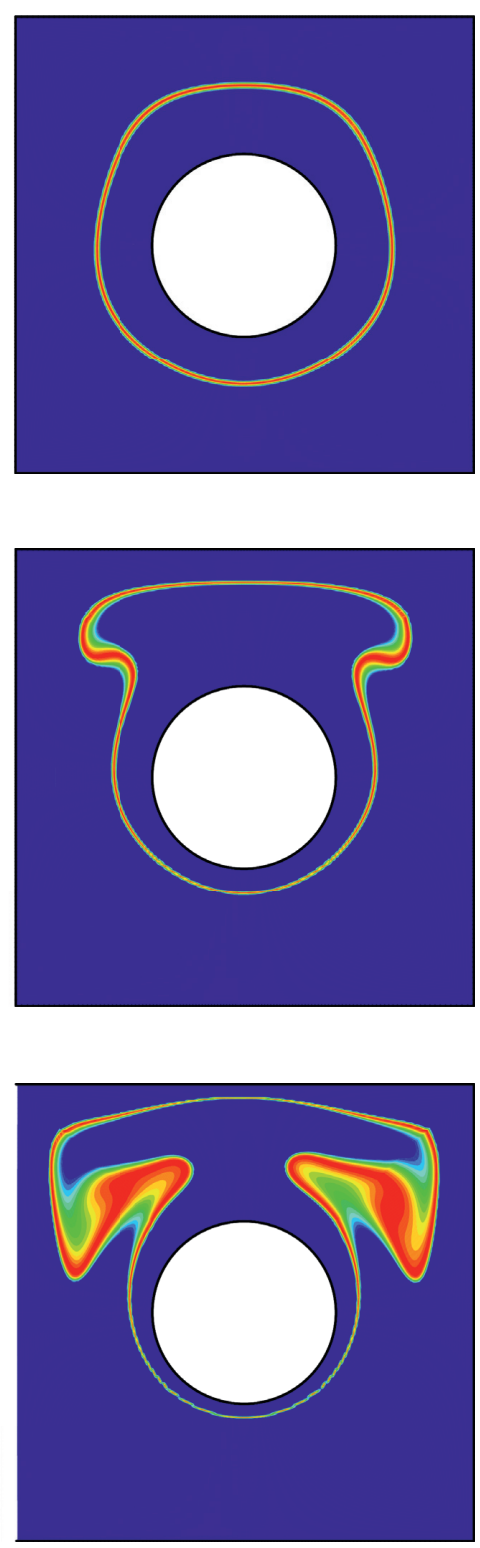

Figure 4: The streamlines, the isotherms, and $\mathrm{Cr}$ contour at $\mathrm{Ra}=10^{4}, 10^{5}$, and $10^{6}$.

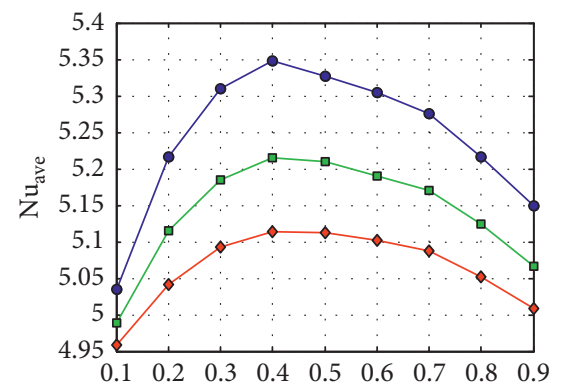

$\theta_{\mathrm{f}}$

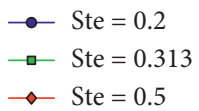

(a)

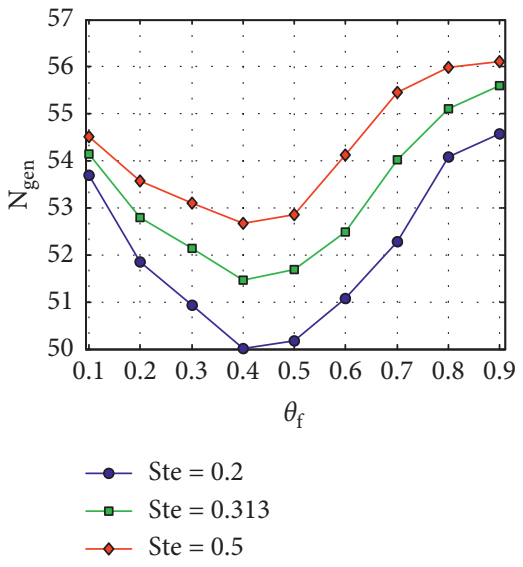

(b)

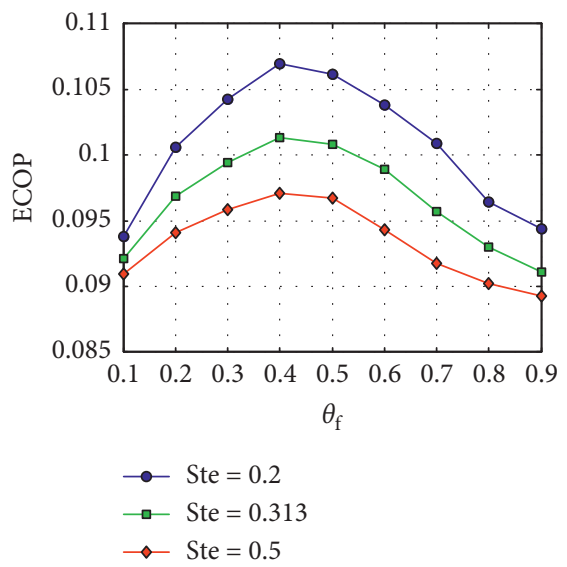

(c)

Figure 5: $\mathrm{Nu}_{\text {ave }}, N_{\text {gen }}$, and ECOP versus the fusion temperature for different values of the Ste at $\operatorname{Ra}=10^{5}$ and $\phi=0.05$. 


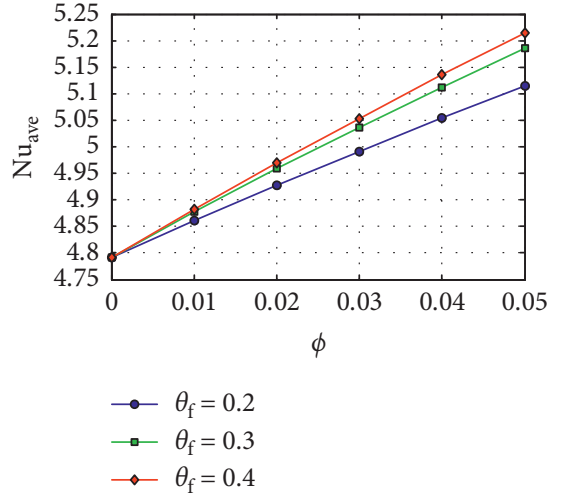

(a)

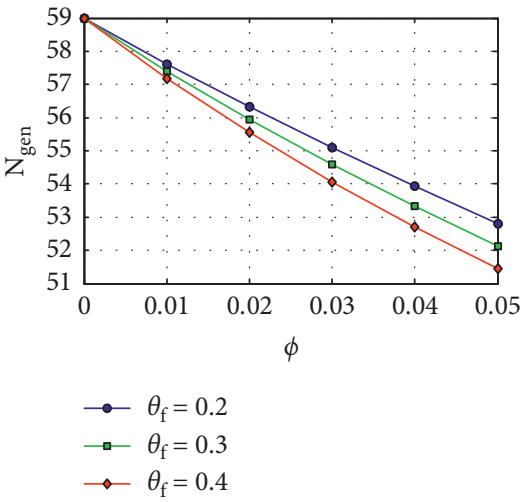

(b)

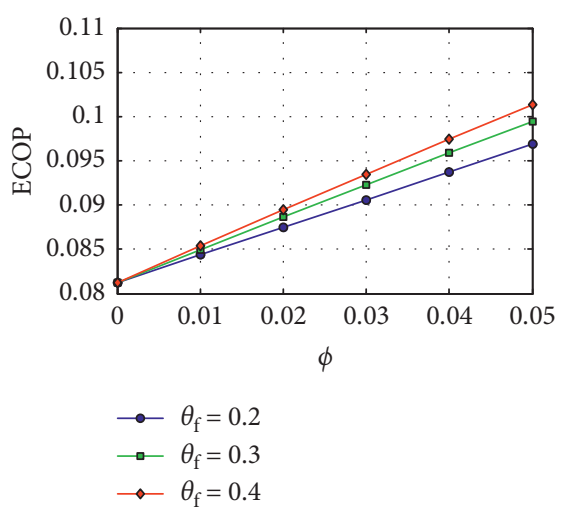

(c)

FIGURE 6: The average Nusselt number, the entropy generation number, and ECOP versus the volume fraction of nanoparticle for three values of fusion temperature.

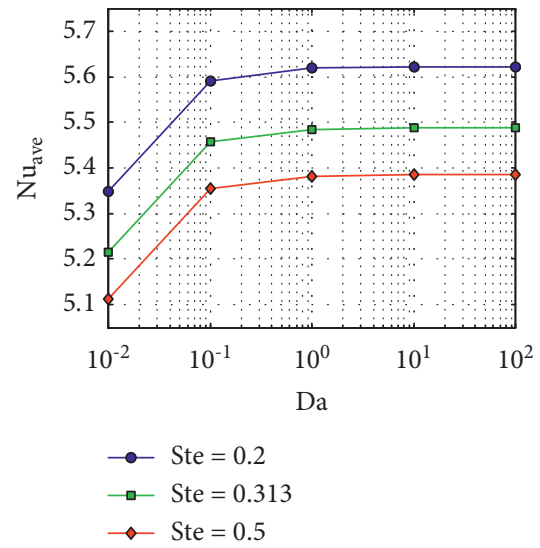

(a)



(b)

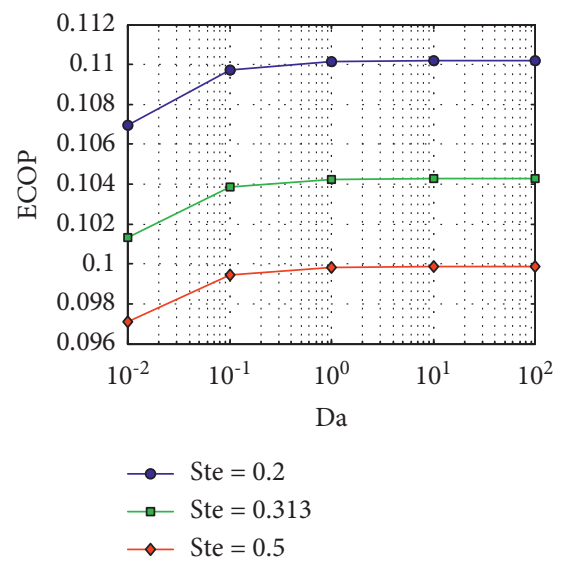

(c)

Figure 7: $\mathrm{Nu}_{\text {ave }}, N_{\text {gen }}$, and ECOP versus the $\mathrm{Da}$ for three values of the Stefan number at $\theta_{f}=0.4$ and $\mathrm{Ra}=10^{5}$.

$25 \%$ to $30 \%$ as $\phi$ increases from 0.01 to 0.05 at $\theta_{f}=0.4$. There is no significant change in $N_{T, \mathrm{PM}}$ with the change in the $\phi$. Besides, the values of $N_{T, \mathrm{HT}}, N_{T, \mathrm{FF}}$, and $N_{T, \mathrm{PM}}$ are approximately constant when $\theta_{f}$ enhances from 0.2 to 0.4 for each value of $\phi$.

Table 5 shows the influence of the effective parameters on $\mathrm{Nu}_{\text {ave }}, N_{\text {gen }}$, and ECOP. The first row in Table 5 is corresponding to the pure fluid $(\phi=0)$. The comparison between the first and second rows discovers that $\mathrm{Nu}_{\text {ave }}$ and ECOP enhance and $N_{\text {gen }}$ reduces when $\phi$ increases from 0 to 0.04 . The comparison between the second and third rows reveals that $\mathrm{Nu}_{\text {ave }}, N_{\text {gen }}$, and ECOP increase with increasing $N_{c}$ (i.e., increasing the thermal conductivity). The comparison between the second and fourth rows displays that $\mathrm{Nu}_{\text {ave }}$ and $N_{\text {gen }}$ decrease, and ECOP increases with increasing $N_{v}$ (i.e., increment the dynamic viscosity). The comparison between the second and fifth rows discovers that $\mathrm{Nu}_{\text {ave }}, N_{\text {gen }}$, and ECOP increase with increasing $N_{c}$ and $N_{v}$, simultaneously. The comparison between the second and sixth rows shows that $\mathrm{Nu}_{\text {ave }}$ and ECOP descend and $N_{\text {gen }}$ ascends when $\lambda$ descends from 0.4 to 0.3 . Hence, the higher values of $\lambda$ are desirable. The comparison between the second and eighth rows demonstrates that $\mathrm{Nu}_{\text {ave }}$ decreases from 5.3568 to $5.3161(0.8 \%$ decrease), $N_{\text {gen }}$ decreases from 54.0578 to $54.9515(1.7 \%$ decrease), and ECOP decreases from 0.0991 to 0.0967 $(2.4 \%$ decrease $)$ when $\theta_{f}$ decreases from 0.52 to 0.22 (57.7\% decreasing). These values indicate that the changes in $\mathrm{Nu}_{\text {ave }}, N_{\text {gen }}$, and ECOP are very small compared to the $\theta_{f}$ changes. The comparison between the second and last rows reveals that $\mathrm{Nu}_{\text {ave }}$ and ECOP ascend and $N_{\text {gen }}$ descends when Ste descends from 0.313 to 0.25 . These numbers indicate that lower values of the Stefan number are desirable. For calculations of Table 5, the decision parameters are $\mathrm{Ra}=10^{5}, \mathrm{AR}=0.2, \mathrm{Da}=0.1, \mathrm{Pr}=6.2$, and $\Phi=10^{-4}$. 
$\mathrm{R} / \mathrm{L}=0.1$

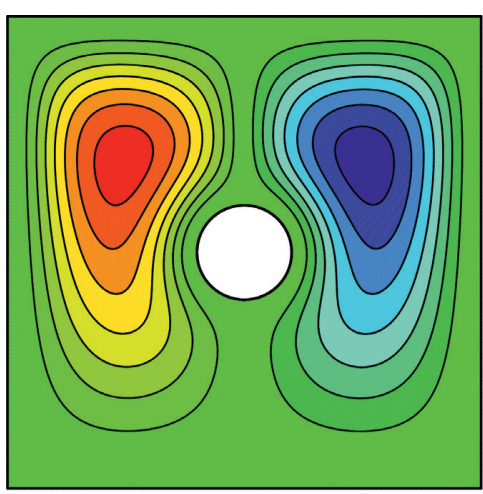

$\left|\Psi_{\max }\right|=7.56$

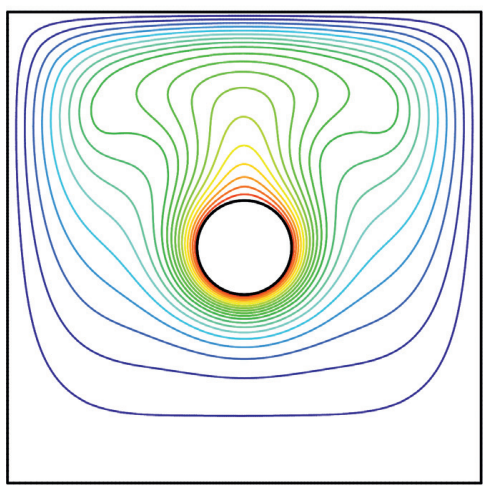

$\mathrm{Nu}_{\text {ave }}=4.3144$

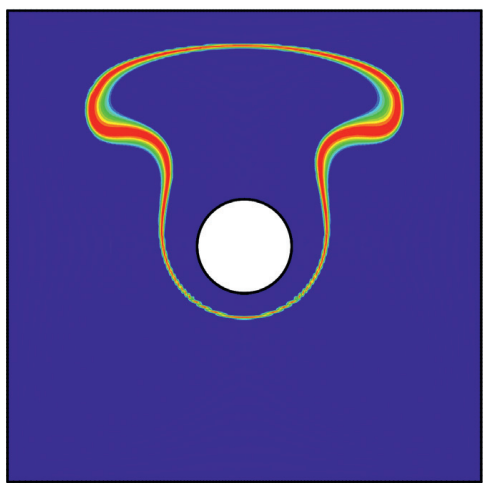

$\mathrm{R} / \mathrm{L}=0.2$

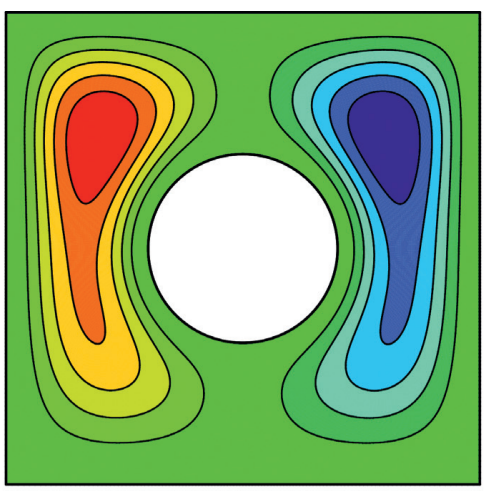

$\left|\Psi_{\max }\right|=6.16$

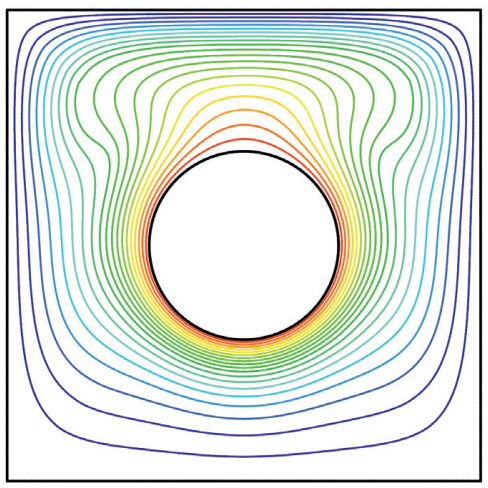

$\mathrm{Nu}_{\text {ave }}=5.6887$

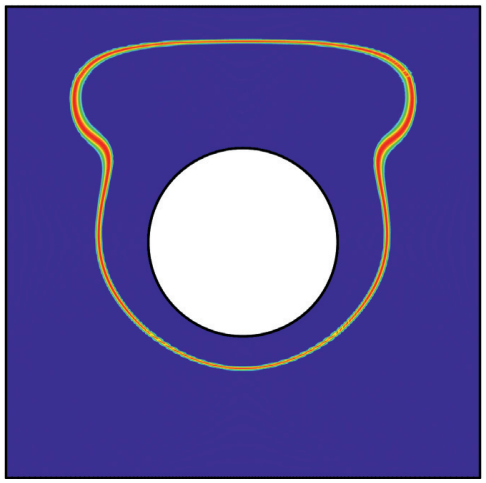

$C r_{\text {max }}=5.99$ and $C r_{\text {min }}=0.97$
$\mathrm{R} / \mathrm{L}=0.3$

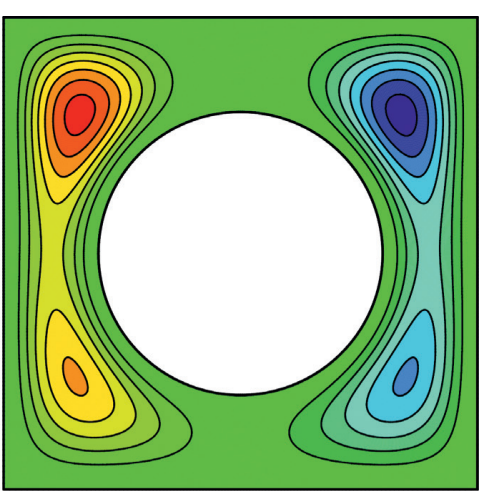

$\left|\Psi_{\max }\right|=3.74$



$\mathrm{Nu}_{\text {ave }}=7.6795$



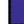

FIgURE 8: The streamlines, the isotherms, and the $\mathrm{Cr}$ contours for various values of $A R$ at $\theta_{f}=0.45$ and $\mathrm{Ra}=10^{5}$.

TABLE 4: The values of $\left|\Psi_{\max }\right|, \mathrm{Nu}_{\text {ave }}$ and $N_{\text {gen }}$ for different values of aspect ratio.

\begin{tabular}{|c|c|c|c|c|c|c|c|c|c|}
\hline \multirow[b]{3}{*}{$\mathrm{Ra}$} & \multirow{2}{*}{\multicolumn{3}{|c|}{$\begin{array}{c}\left|\Psi_{\max }\right| \\
A R\end{array}$}} & \multicolumn{3}{|c|}{$\mathrm{Nu}_{\mathrm{ave}}$} & \multicolumn{3}{|c|}{$N_{\text {gen }}$} \\
\hline & & & & & $A R$ & & & $A R$ & \\
\hline & 0.1 & 0.2 & 0.3 & 0.1 & 0.2 & 0.3 & 0.1 & 0.2 & 0.3 \\
\hline 0 & - & - & - & 2.3789 & 4.0922 & 6.9556 & 8.2697 & 11.9824 & 23.2093 \\
\hline $10^{4}$ & 1.039 & 0.6475 & 0.349 & 2.4934 & 4.1342 & 6.9692 & 9.3995 & 12.5937 & 23.5294 \\
\hline $10^{5}$ & 7.558 & 6.155 & 3.740 & 4.3144 & 5.6887 & 7.6795 & 51.6553 & 54.6358 & 61.9543 \\
\hline
\end{tabular}




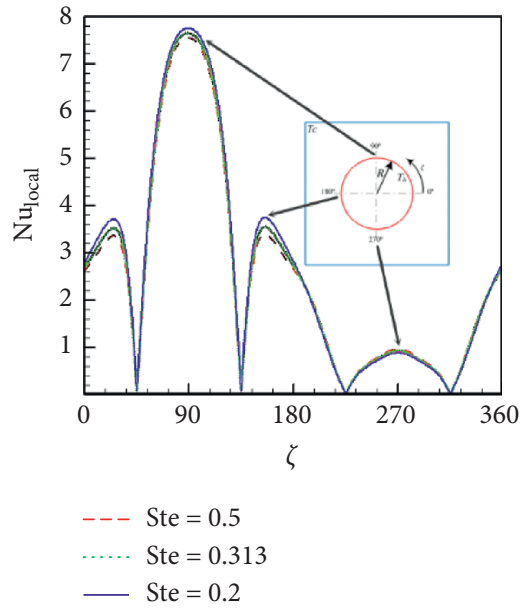

(a)

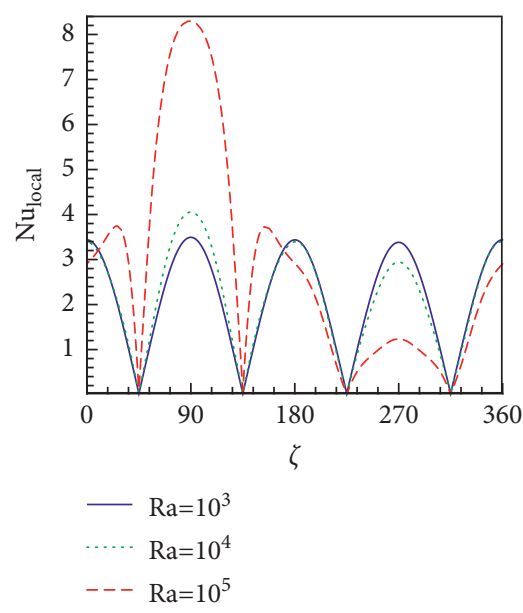

(b)

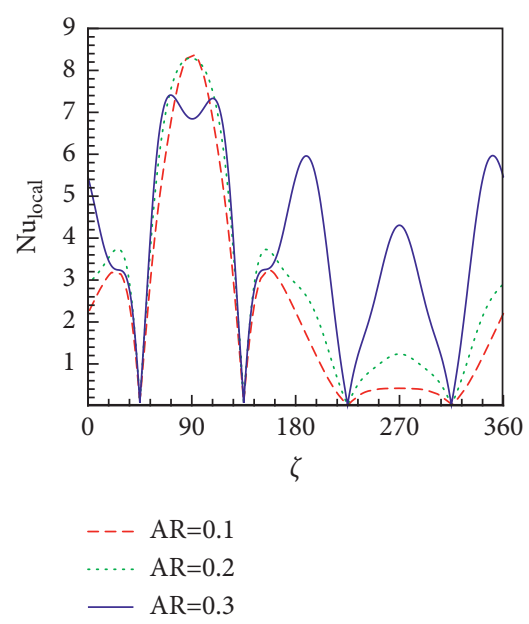

(c)

Figure 9: Variations of the local Nusselt number versus the rotation angle for three values of (a) the Stefan number, (b) The Rayleigh number, and (c) the aspect ratio.

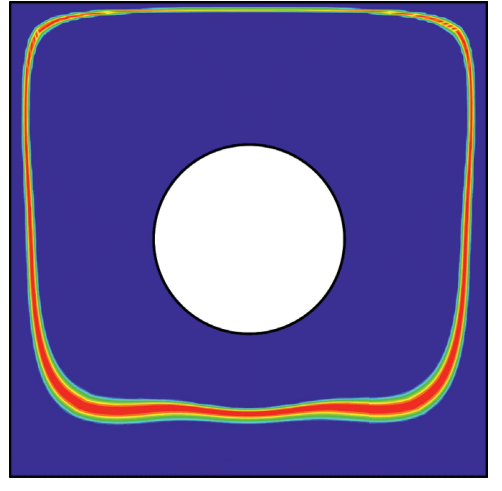

(a)

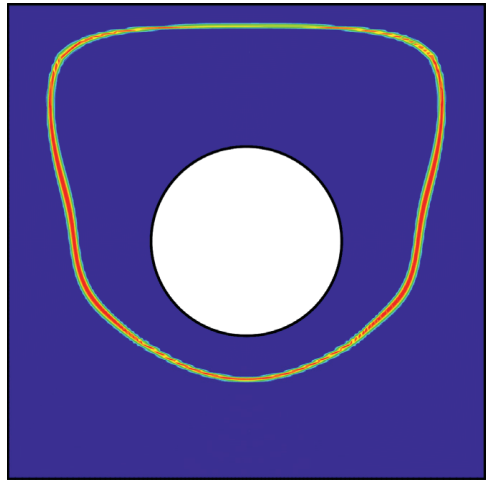

(b)

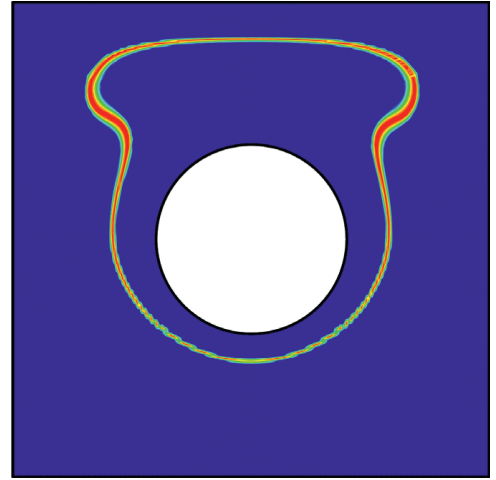

(c)

Figure 10: The Cr contours for three values of the nondimensional fusion temperature. (a) $\theta_{f}=0.1$, (b) $\theta_{f}=0.3$, and (c) $\theta_{f}=0.5$.

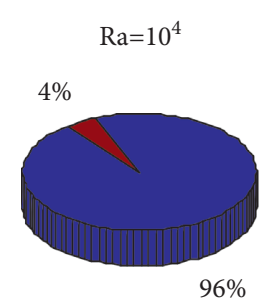

$\mathrm{Ra}=10^{5}$

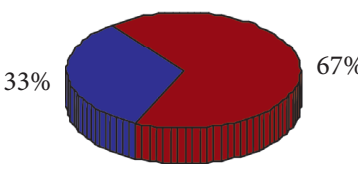

$\mathrm{N}_{\mathrm{T}, \mathrm{HT}}$
$\mathrm{Ra}=5 \times 10^{4}$

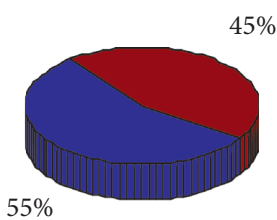

$\mathrm{Ra}=5 \times 10^{5}$

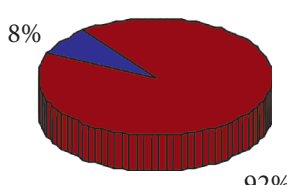

92\% $\theta_{\mathrm{f}}=0.2$ and $\phi=0.01$

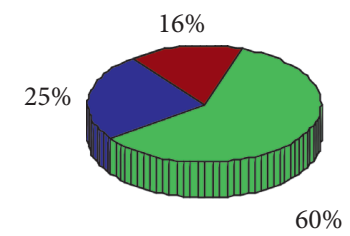

$\theta_{\mathrm{f}}=0.4$ and $\phi=0.01$

$16 \%$

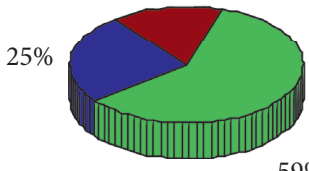

$59 \%$

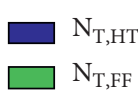

$\square \mathrm{N}_{\mathrm{T}, \mathrm{PM}}$ $\theta_{\mathrm{f}}=0.2$ and $\phi=0.05$

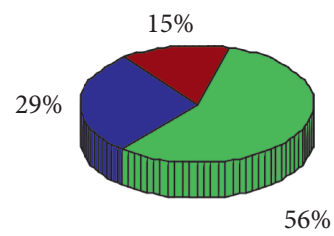

$\theta_{\mathrm{f}}=0.4$ and $\phi=0.05$

$15 \%$

$30 \%$

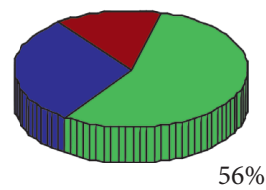

(a)

(b)

Figure 11: Contribution of components in the total entropy generation number (a) for different values of Ra and (b) for two values of $\phi$ and $\theta_{f}$. 
TABLE 5: The effect of active parameters on $\mathrm{Nu}_{\text {ave }}, N_{\text {gen }}$, and ECOP.

\begin{tabular}{|c|c|c|c|c|c|c|c|c|c|c|c|}
\hline No. & $\theta_{f}$ & $\phi$ & $\lambda$ & $\rho_{p} / \rho_{f}$ & Ste & $\delta$ & $N_{c}$ & $N_{v}$ & $\mathrm{Nu}_{\text {ave }}$ & $N_{\text {gen }}$ & ECOP \\
\hline 1 & 0.52 & 0.00 & 0.4 & 0.9 & 0.313 & 0.05 & 3 & 3 & 5.0186 & 59.6706 & 0.0841 \\
\hline 2 & 0.52 & 0.04 & 0.4 & 0.9 & 0.313 & 0.05 & 3 & 3 & 5.3568 & 54.0578 & 0.0991 \\
\hline 3 & 0.52 & 0.04 & 0.4 & 0.9 & 0.313 & 0.05 & 6 & 3 & 5.7550 & 57.1648 & 0.1007 \\
\hline 4 & 0.52 & 0.04 & 0.4 & 0.9 & 0.313 & 0.05 & 3 & 6 & 5.1998 & 51.6790 & 0.1006 \\
\hline 5 & 0.52 & 0.04 & 0.4 & 0.9 & 0.313 & 0.05 & 6 & 6 & 5.5889 & 54.5611 & 0.1024 \\
\hline 6 & 0.52 & 0.04 & 0.3 & 0.9 & 0.313 & 0.05 & 3 & 3 & 5.3510 & 54.1049 & 0.0989 \\
\hline 7 & 0.52 & 0.04 & 0.4 & 0.7 & 0.313 & 0.05 & 3 & 3 & 5.3441 & 53.5620 & 0.0998 \\
\hline 8 & 0.22 & 0.04 & 0.4 & 0.9 & 0.313 & 0.05 & 3 & 3 & 5.3161 & 54.9515 & 0.0967 \\
\hline 9 & 0.52 & 0.04 & 0.4 & 0.9 & 0.25 & 0.05 & 3 & 3 & 5.4033 & 53.3842 & 0.1012 \\
\hline
\end{tabular}

\section{Conclusion}

In this work, entropy generation and free convection were investigated in a complex cavity filled with NEPCMs. The nondimensional governing equations were solved by CVFEM. The CVFEM method could properly determine the behavior of the fluid in the understudy cavity. In future work, the effect of NEPCM suspension can be compared with nanofluids in an exclusive property like non-Newtonian fluid in a complicated geometry by considering the effect of some other fields and parameters. The important highlights can be summarized as follows:

(i) The values of $\left|\Psi_{\max }\right|$ and $\mathrm{Nu}_{\text {ave }}$ increase when the Rayleigh number ascends. The values of $\left|\Psi_{\max }\right|$ are $0.804,6.81$, and 21.64, and the values of $\mathrm{Nu}_{\text {ave }}$ are $4.1636,6.0981$, and 10.962 , for $\mathrm{Ra}=10^{4}, 10^{5}$ and $10^{6}$, respectively (Figure 4 ).

(ii) There is a maximum value for $\mathrm{Nu}_{\text {ave }}$ and a minimum value for $\mathrm{Nu}_{\text {ave }}$ at $\theta_{f}=0.4$ for each value of the Stefan number (Figure 5).

(iii) $\mathrm{Nu}_{\text {ave }}$ increases by $8.8 \%$ while $N_{\text {gen }}$ decreases by $12.8 \%$ when $\phi$ goes up $5 \%$ at $\theta_{f}=0.4$ (Figure 6).

(iv) $\mathrm{Nu}_{\text {ave }}, N_{\text {gen }}$, and ECOP increase by $5.3 \%, 2.4 \%$, and $2.9 \%$, respectively, as thr Darcy number goes up from 0.01 to 100 at Ste $=0.5$ (Figure 7).

(v) The values of $\left|\Psi_{\max }\right|$ decrease while the values of $\mathrm{Nu}_{\text {ave }}$ and $N_{\text {gen }}$ increase with increasing the values of aspect ratio. The values of $\left|\Psi_{\max }\right|$ descend $50.52 \%$, whereas $\mathrm{Nu}_{\text {ave }}$ and $N_{\text {gen }}$ ascend by $78 \%$ and $19.94 \%$, respectively, when $A R$ ascends from 0.1 to 0.3 at $\mathrm{Ra}=10^{5}$ (Figure 8 and Table 4).

(vi) There is a maximum $\mathrm{Nu}_{\text {local }}$ at the top of the hot wall $\left(\zeta=90^{\circ}\right)$ for each value of the Stefan number (Figure 9).

(vii) The red region is similar to a square (cold wall) at $\theta_{f}=0.1$. It is similar to an irregular ring at $\theta_{f}=0.3$, while it is similar to a plume at $\theta_{f}=0.5$ (Figure 10).

(viii) $N_{T, \mathrm{HT}}$ goes up from $25 \%$ to $30 \%$ as $\phi$ increases from 0.01 to 0.05 at $\theta_{f}=0.4$ (Figure 11).

(ix) The values of $\mathrm{Nu}_{\text {ave }}, N_{\text {gen }}$, and ECOP increase with ascending $N_{c}$, but the values of $\mathrm{Nu}_{\text {ave }}$ and $N_{\text {gen }}$ decrease and ECOP increases with increasing $N_{v}$ (Table 5).

\section{Nomenclature}

$C_{p}: \quad$ Specific heat at constant pressure $\left(\mathrm{J} \mathrm{kg}^{-1} \mathrm{~K}^{-1}\right)$

Cr: The heat capacity ratio

$f: \quad$ The nondimensional fusion function

Da: Darcy number

ECOP: Ecological coefficient of performance

g: $\quad$ Gravitational acceleration $\left(\mathrm{ms}^{-2}\right)$

$k: \quad$ Thermal conductivity $\left(\mathrm{Wm}^{-1} \mathrm{~K}^{-1}\right)$

K: $\quad$ Permeability of the medium $\left(\mathrm{m}^{2}\right)$

$L: \quad$ Gap between the inner and outer walls of the enclosure $(\mathrm{m})$

$N_{\text {gen }}: \quad$ Entropy generation number

$N c$ : Thermal conductivity parameter

$\mathrm{Nu}$ : Nusselt number

$N v: \quad$ Viscosity parameter

Pr: Prandtl number

Ra: Rayleigh number

Ste: $\quad$ Stefan number

$\dot{S}_{\text {gen }}: \quad$ Rate of entropy generation per unit volume $\left(\mathrm{J} \mathrm{s}^{-1}\right.$ $\mathrm{K}^{-1} \mathrm{~m}^{-3}$ )

$T: \quad$ Temperature $(\mathrm{K})$

$T_{0}$ : $\quad$ Mean temperature (K)

$u, v: \quad$ Components of velocity $\left(\mathrm{m} \mathrm{s}^{-1}\right)$

$X, Y: \quad$ Dimensionless coordinates.

$\alpha: \quad$ Thermal diffusivity $\left(\mathrm{m}^{2} \mathrm{~s}^{-1}\right)$

$\beta: \quad$ Thermal expansion coefficient $\left(\mathrm{K}^{-1}\right)$

$\delta: \quad$ Nondimensional parameter of fusion range

$\Theta: \quad$ Dimensionless temperature

$\zeta: \quad$ Rotation angle $\left({ }^{\circ}\right)$

$\lambda$ : $\quad$ Ratio of the heat capacity of the NEPCM nanoparticles to the base fluid

$\mu$ : $\quad$ Dynamic viscosity $\left(\mathrm{N} \mathrm{s} \mathrm{m}^{-2}\right)$

$\nu: \quad$ Kinematic viscosity $\left(\mathrm{m}^{2} \mathrm{~s}^{-1}\right)$

$\rho: \quad$ Density $\left(\mathrm{kg} \mathrm{m}^{-3}\right)$

$\Delta T: \quad$ Temperature difference $(\mathrm{K})\left(\Delta T=T_{h}-T_{c}\right)$

$\phi: \quad$ Volume fraction of NEPCM nanoparticles

$\Phi: \quad$ Irreversibility distribution ratio

$\Psi: \quad$ Dimensionless stream function

$\Omega: \quad$ Dimensionless vorticity

ave: Average

$b$ : $\quad$ Bulk properties of the suspension

c: $\quad$ Cold

$f$ : $\quad$ Fluid

FF: Fluid friction

gen: Generation 
$\begin{array}{ll}h: & \text { Hot } \\ \text { HT: } & \text { Heat transfer } \\ L: & \text { Local } \\ \text { PM: } & \text { Porous medium. }\end{array}$

\section{Data Availability}

The data used to support the findings of this study are available from the corresponding author upon request.

\section{Conflicts of Interest}

The authors declare that they have no conflicts of interest.

\section{References}

[1] M. A. Sheremet, D. S. Cimpean, and I. Pop, "Free convection in a partially heated wavy porous cavity filled with a nanofluid under the effects of Brownian diffusion and thermophoresis," Applied Thermal Engineering, vol. 113, pp. 413-418, 2017.

[2] M. Hashemi-Tilehnoee, A. S. Dogonchi, S. M. Seyyedi, and M. Sharifpur, "Magneto-fluid dynamic and second law analysis in a hot porous cavity filled by nanofluid and nanoencapsulated phase change material suspension with different layout of cooling channels," Journal of Energy Storage, vol. 31, p. $101720,2020$.

[3] A. S. Dogonchi, M. Waqas, S. M. Seyyedi, M. HashemiTilehnoee, and D. D. Ganji, "CVFEM analysis for $\mathrm{Fe}_{3} \mathrm{O}_{4}-\mathrm{H}_{2} \mathrm{O}$ nanofluid in an annulus subject to thermal radiation," International Journal of Heat and Mass Transfer, vol. 132, pp. $473-483,2019$.

[4] S. M. Seyyedi, A. S. Dogonchi, M. Hashemi-Tilehnoee, M. Waqas, and D. D. Ganji, "Entropy generation and economic analyses in a nanofluid filled L-shaped enclosure subjected to an oriented magnetic field," Applied Thermal Engineering, vol. 168, p. 114789, 2020.

[5] M. Hashemi-Tilehnoee, S. Tashakor, A. S. Dogonchi, S. M. Seyyedi, and M. Khaleghi, "Entropy generation in concentric annuli of $400 \mathrm{kV}$ gas-insulated transmission line," Thermal Science and Engineering Progress, vol. 19, p. 100614, 2020.

[6] S. M. Seyyedi, A. S. Dogonchi, M. Hashemi-Tilehnoee, D. D. Ganji, and A. J. Chamkha, "Second law analysis of magneto-natural convection in a nanofluid filled wavy-hexagonal porous enclosure," International Journal of Numerical Methods for Heat and Fluid Flow, vol. 30, no. 11, pp. 48114836, 2020.

[7] M. Hashemi-Tilehnoee, A. S. Dogonchi, S. M. Seyyedi, A. J. Chamkha, and D. D. Ganji, "Magnetohydrodynamic natural convection and entropy generation analyses inside a nanofluid filled incinerator-shaped porous cavity with wavy heater block," Journal of Thermal Analysis and Calorimetry, vol. 1, pp. 1-13, 2020.

[8] S. M. Seyyedi, M. Hashemi-Tilehnoee, and M. Sharifpur, "Effect of inclined magnetic field on the entropy generation in an annulus filled with NEPCM suspension," Mathematical Problems in Engineering, vol. 2021, Article ID 8103300, 2021.

[9] Z. Ling, Z. Zhang, G. Shi et al., "Review on thermal management systems using phase change materials for electronic components, Li-ion batteries and photovoltaic modules," Renewable and Sustainable Energy Reviews, vol. 31, pp. 427438, 2014.
[10] S. Gharbi, S. Harmand, and S. B. Jabrallah, "Experimental comparison between different configurations of PCM based heat sinks for cooling electronic components," Applied Thermal Engineering, vol. 87, pp. 454-462, 2015.

[11] S. M. Shalaby, M. A. Bek, and A. A. El-Sebaii, "Solar dryers with PCM as energy storage medium: a review," Renewable and Sustainable Energy Reviews, vol. 33, pp. 110-116, 2014.

[12] F. Agyenim, "The use of enhanced heat transfer phase change materials $(\mathrm{PCM})$ to improve the coefficient of performance (COP) of solar powered $\mathrm{LiBr} / \mathrm{H} 2 \mathrm{O}$ absorption cooling systems," Renewable Energy, vol. 87, pp. 229-239, 2016.

[13] N. Maruoka and T. Akiyama, "Thermal stress analysis of PCM encapsulation for heat recovery of high temperature waste heat," Journal of Chemical Engineering of Japan, vol. 36, no. 7, pp. 794-798, 2003.

[14] T. Nomura, N. Okinaka, and T. Akiyama, "Waste heat transportation system, using phase change material (PCM) from steelworks to chemical plant," Resources, Conservation and Recycling, vol. 54, no. 11, pp. 1000-1006, 2010.

[15] S. Tiari, S. Qiu, and M. Mahdavi, "Numerical study of finned heat pipe-assisted thermal energy storage system with high temperature phase change material," Energy Conversion and Management, vol. 89, pp. 833-842, 2015.

[16] S. Tiari, S. Qiu, and M. Mahdavi, "Discharging process of a finned heat pipe-assisted thermal energy storage system with high temperature phase change material," Energy Conversion and Management, vol. 118, pp. 426-437, 2016.

[17] M. Ghalambaz, A. J. Chamkha, and D. Wen, "Natural convective flow and heat transfer of nano-encapsulated phase change materials (NEPCMs) in a cavity," International Journal of Heat and Mass Transfer, vol. 138, pp. 738-749, 2019.

[18] H. Lu, H. R. Seyf, Y. Zhang, and H. B. Ma, "Heat transfer enhancement of backward-facing step flow by using nanoencapsulated phase change material slurry," Numerical Heat Transfer, Part A: Applications, vol. 67, no. 4, pp. 381-400, 2015.

[19] C. Liu, Z. Rao, J. Zhao, Y. Huo, and Y. Li, "Review on nanoencapsulated phase change materials: preparation, characterization and heat transfer enhancement," Nanomaterials and Energy, vol. 13, pp. 814-826, 2015.

[20] L. Chai, R. Shaukat, L. Wang, and H. Sheng Wang, "A review on heat transfer and hydrodynamic characteristics of nano/ microencapsulated phase change slurry (N/MPCS) in mini/ microchannel heat sinks," Applied Thermal Engineering, vol. 135, 2018.

[21] M. Ghalambaz, T. Groşan, and I. Pop, "Mixed convection boundary layer flow and heat transfer over a vertical plate embedded in a porous medium filled with a suspension of nano-encapsulated phase change materials," Journal of Molecular Liquids, vol. 293, p. 111432, 2019.

[22] H. Ahmad, S. A. M. Mehryan, and M. Ghalambaz, "Time periodic natural convection heat transfer in a nano-encapsulated phase-change suspension," International Journal of Mechanical Sciences, vol. 166, 2019.

[23] M. Ghalambaz, S. A. M. Mehryan, H. Ahmad, and V. Ali, "Unsteady natural convection flow of a suspension comprising nano-encapsulated phase change materials (NEPCMs) in a porous medium," Advanced Powder Technology, vol. 31, no. 3, 2020.

[24] M. F. Md Basir, K. Naganthran, E. Azhar et al., "Unsteady nano-bioconvective channel flow with effect of $n$th order chemical reaction," Open Physics, vol. 18, no. 1, pp. 1011-1024, 2020.

[25] S. A. M. Mehryan, M. Ghalambaz, L. Sasani Gargari, A. Hajjar, and M. Sheremet, "Natural convection flow of a suspension 
containing nano-encapsulated phase change particles in an eccentric annulus," Journal of Energy Storage, vol. 28, p. 101236, 2020.

[26] C. J. Ho, Y.-C. Liu, M. Ghalambaz, and W.-M. Yan, "Forced convection heat transfer of Nano-Encapsulated Phase Change Material (NEPCM) suspension in a mini-channel heatsink," International Journal of Heat and Mass Transfer, vol. 155, p. $119858,2020$.

[27] S. M. Hashem Zadeh, S. A. M. Mehryan, M. Sheremet, M. Ghodrat, and M. Ghalambaz, "Thermo-hydrodynamic and entropy generation analysis of a dilute aqueous suspension enhanced with nano-encapsulated phase change material," International Journal of Mechanical Sciences, vol. 178, p. $105609,2020$.

[28] S. M. Hashem Zadeh, S. A. M. Mehryan, M. S. Islam, and M. Ghalambaz, "Irreversibility analysis of thermally driven flow of a water-based suspension with dispersed nano-sized capsules of phase change material," International Journal of Heat and Mass Transfer, vol. 155, p. 119796, 2020.

[29] F. Moukalled and S. Acharya, "Natural convection in the annulus between concentric horizontal circular and square cylinders," Journal of Thermophysics and Heat Transfer, vol. 10, no. 3, 1996.

[30] B. S. Kim, D. S. Lee, M. Y. Ha, and H. S. Yoon, "A numerical study of natural convection in a square enclosure with a circular cylinder at different vertical locations," International Journal of Heat and Mass Transfer, vol. 51, pp. 1888-1906, 2008 . 